\title{
Estresse discente em um curso de Fonoaudiologia
}

\author{
Student stress in a speech, language and hearing sciences
}

\section{undergraduate course}

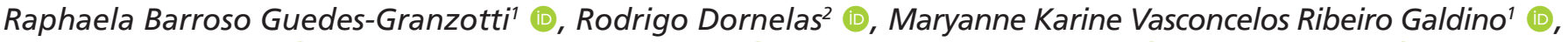 \\ Ingrid Santos Leite ${ }^{1}$ (D), Priscila Feliciano de Oliveira ${ }^{1}$ (D), Patrícia Pinatti Moreira ${ }^{3}$ (D), Kelly da Silva ${ }^{3}$ (1)
}

\begin{abstract}
RESUMO
Objetivo: verificar a prevalência de estresse e os fatores associados à sua presença em discentes de um curso de Fonoaudiologia. Métodos: participaram 105 discentes, maiores de 18 anos, de ambos os sexos, matriculados no curso de Fonoaudiologia de uma universidade federal. Foram excluídos os discentes que não estavam com a matrícula ativa durante o período da pesquisa. Todos responderam a um questionário sociodemográfico e ao Inventário de Sintomatologia de Stress de Lippi, que identifica os sintomas de estresse, bem como a sua respectiva fase e os sintomas predominantes. Os dados receberam tratamento estatístico, sendo estipulado o nível de significância de $5 \%$. Resultados: dentre os participantes, $77,1 \%$ apresentaram resultados sugestivos de estresse, sendo que, destes, $77,7 \%$ estavam na fase de "resistência", 18,5\% na fase de "quase exaustão"; $2,5 \%$ na fase de "alerta"; e 1,2\% na fase de "exaustão". Quanto à sintomatologia $64,2 \%$ apresentaram predominância de sintomas psicológicos, 18,5\%, físicos e 17,3\%, físicos e psicológicos. A análise estatística mostrou a presença significativamente superior de estresse entre as mulheres e entre os que não trabalhavam e a relação do estresse com o ano de estudo. Conclusão: um elevado número de discentes de Fonoaudiologia foi identificado com estresse, sendo a fase de "resistência" a mais frequente, com prevalência de sintomas psicológicos. Os fatores associados ao estresse foram sexo, com maior prejuízo entre as mulheres, o fato de não exercerem atividade remunerada e o ano do curso em que estavam matriculados, sendo que no primeiro ano foi observada menor prevalência de estresse e no último ano, maior.
\end{abstract}

Palavras-chave: Estresse fisiológico; Saúde mental; Promoção da saúde; Educação superior; Fonoaudiologia

\begin{abstract}
Purpose: To verify the prevalence and factors associated with the presence of stress in students of a Speech, Language, and Hearing Sciences course. Methods: 105 students participated, over 18 years old, of both sexes, enrolled in the Speech, Language and Hearing Sciences course at a Federal University. Students who were not active during participation in the research were excluded. All respond to a sociodemographic questionnaire and Lipp's Stress Symptoms Inventory, which identifies the symptoms of stress, as well as their specific phase and the predominant symptoms. The data received statistical treatment being stipulated or significance level of 5\%. Results: $77.1 \%$ showed results suggestive of stress, of which $77.7 \%$ were in the "resistance" phase, $18.5 \%$ in the "almost exhaustion"; $2.5 \%$ in the "alarm"; and $1.2 \%$ in the "exhaustion". As for the symptomatology, $64.2 \%$ had a predominance of psychological symptoms, $18.5 \%$ physical, and $17.3 \%$ physical and psychological. The statistical analysis showed a significantly higher presence of stress among women and among those who do not work. As well as a relationship between stress and the study year. Conclusion: A high number of students were identified with stress, with the resistance phase being the most frequent with psychological predominance. The factors associated with stress were gender, with the greatest loss in women, in people who do not engage in paid work, and the year of the course in which they are enrolled, with a lower prevalence of stress being observed in the first year and a higher year in the last year.
\end{abstract}

Keywords: Stress physiological; Mental health; Health promotion; Education higher; Speech, Language and Hearing Sciences

\footnotetext{
Trabalho realizado no Curso de Fonoaudiologia, Universidade Federal de Sergipe - UFS - São Cristóvão (SE), Brasil.

${ }^{1}$ Departamento de Fonoaudiologia, Universidade Federal de Sergipe - UFS, Cidade Universitária Prof. José Aloísio de Campos - São Cristóvão (SE), Brasil.

${ }^{2}$ Departamento de Fonoaudiologia, Universidade Federal do Rio de Janeiro - UFRJ - Rio de Janeiro (RJ), Brasil.

${ }^{3}$ Departamento de Fonoaudiologia da Universidade Federal de Sergipe - UFS, Campus Professor Antônio Garcia Filho - Lagarto (SE), Brasil.

Conflito de interesses: Não.

Contribuição dos autores: RBGG participou, na condição de orientador, da idealização do estudo, análise, interpretação dos dados e redação do artigo; MKVRG e ISL participaram da coleta e redação do artigo; RD, PFO, PPM e KS participaram da análise e interpretação dos dados e redação do artigo.

Financiamento: Nada a declarar.

Autor correspondente: Raphaela Barroso Guedes-Granzotti. E-mail: raphaelabgg@gmail.com

Recebido: Maio 08, 2020; Aceito: Setembro 04, 2020
} 


\section{INTRODUÇÃO}

O termo estresse foi utilizado pela primeira vez no século XVII para descrever um complexo fenômeno composto de tensão-angústia-desconforto ${ }^{(1)}$. Seguindo um panorama multidimensional, o estresse pode ser descrito tanto como uma resposta fisiológica, como psicológica, desencadeada por um estímulo estressor que exija esforço de uma função além de sua capacidade de adaptação ou tolerância ${ }^{(2)}$. A forma de (re)agir ao estresse está relacionada a fatores chamados moderadores, que são a personalidade, o nível educacional, o fator social, entre outros ${ }^{(1,2)}$.

A teoria trifásica da Síndrome da Adaptação Geral $(\mathrm{SAG})^{(3)}$ foi reformulada ${ }^{(4)}$ com a identificação de mais uma fase (quase exaustão"), tornando-a um modelo quadrifásico (estágio de "alerta", de "resistência", de "quase exaustão" e de "exaustão"). O estágio de "alerta", fase positiva do estresse, é ativado quando o indivíduo é confrontado com uma situação crítica repentina e ocorre uma alta produção de adrenalina, fundamental para a sobrevivência da espécie. O estágio de "resistência" é a tentativa de reequilíbrio físico e mental, em que o organismo altera seus parâmetros de normalidade e concentra a reação interna em um determinado órgão-alvo. Caso ocorra persistência na exposição ao agente estressor, pode haver uma ruptura na resistência do indivíduo, fazendo com que ele entre na fase de "quase exaustão", iniciando o processo de adoecimento. O estágio de "exaustão" é aquele em que o excesso de atividades e o alto consumo de energia impossibilitam ao indivíduo manter-se física e psicologicamente bem para a realização das atividades diárias $^{(4)}$.

Uma fase frequentemente associada à ocorrência de estresse é a universitária, principalmente em estudantes da área da saúde ${ }^{(5-9)}$. $\mathrm{O}$ ingresso na faculdade traz consigo muitas mudanças na rotina, que repercutem na qualidade de vida e na saúde, e os eventos estressores mais citados por universitários se referem à dúvida na escolha do curso, problemas no convívio social, distância dos familiares, problemas financeiros e responsabilidade quanto à formação profissional ${ }^{(5,10,11)}$.

Todas essas adversidades podem gerar reações fisiológicas e se não houver uma adaptação aos agentes estressores as reações oriundas do estresse podem fazer parte da vida dos universitários ${ }^{(10,11)}$, comprometendo o raciocínio, memorização e motivação no processo de aprendizagem ${ }^{(5)}$. Considerando as diversas implicações do estresse entre universitários e a relevância do tema, o objetivo deste estudo foi verificar a prevalência de estresse e os fatores associados à sua presença em discentes de um curso de Fonoaudiologia.

\section{MÉTODOS}

Estudo transversal, quantitativo, observacional e analítico, seguindo as recomendações éticas vigentes (parecer $\mathrm{n}^{0} 3.377 .652$ do Comitê de Ética em Pesquisa - CEP - Universidade Federal de Sergipe).

Participaram discentes de Fonoaudiologia de uma universidade pública, de ambos os sexos, que estavam com a matrícula ativa durante o período da pesquisa (primeiro semestre de 2019).

A amostra constituída de 105 discentes, 24 (22,9\%) do sexo masculino e $81(77,1 \%)$ do feminino, apresentou grau de confiabilidade de $95 \%$ e margem de erro menor que $5 \%$, considerando um total de 204 discentes ativos no curso.

Todos responderam a questões de identificação e perfil sociodemográfico e ao Inventário de Sintomas de Stress para Adultos de Lipp (ISSL) ${ }^{(4)}$, em sala silente, climatizada e iluminada. O ISSL é autoaplicável, fornece dados objetivos a respeito da presença e fase correspondente do estresse e a predominância dos sintomas.

Por se tratar de uma amostra com distribuição normal, foi utilizado, para comparação do número de discentes com estresse nos quatro períodos, o teste Anova de uma via, seguido pelo teste de Post Hoc de Tukey. Para a associação entre os resultados qualitativos, foi utilizado o teste Qui-quadrado, sendo considerado o nível de significância de 5\%.

\section{RESULTADOS}

A grande maioria dos universitários era do sexo feminino e não exercia atividade remunerada; houve equilíbrio quanto à formação no ensino médio, assim como quanto ao recebimento de bolsas ou auxílios da universidade (Tabela 1).

Tabela 1. Perfil dos discentes participantes da pesquisa por ano de estudo

\begin{tabular}{|c|c|c|c|c|c|c|c|c|c|c|}
\hline & \multirow{3}{*}{$\mathrm{N}^{\circ}$ part. } & \multirow{3}{*}{$\begin{array}{c}\text { Média de } \\
\text { idade } \\
\text { 土DP }\end{array}$} & \multicolumn{2}{|c|}{ Sexo } & \multicolumn{2}{|c|}{$\begin{array}{c}\text { Formação no ensino } \\
\text { médio }\end{array}$} & \multicolumn{2}{|c|}{ Atividade remunerada } & \multicolumn{2}{|c|}{$\begin{array}{c}\text { Bolsa ou auxílio da } \\
\text { universidade }\end{array}$} \\
\hline & & & M & $\mathbf{F}$ & Pública & Privada & Sim & Não & Sim & Não \\
\hline & & & n (\%) & n (\%) & n (\%) & n (\%) & n (\%) & n (\%) & n (\%) & n (\%) \\
\hline $1^{\circ}$ & 12 & 20,7 & 4 & 8 & 7 & 5 & 2 & 10 & 3 & 9 \\
\hline ano & $(11,4 \%)$ & $\pm 8,1$ & $(33,3 \%)$ & $(66,7 \%)$ & $(58,3 \%)$ & $(41,7 \%)$ & $(16,7 \%)$ & $(83,3 \%)$ & $(25 \%)$ & $(75 \%)$ \\
\hline $2^{\circ}$ & 27 & 22,4 & 6 & 21 & 14 & 13 & 4 & 23 & 15 & 12 \\
\hline ano & $(25,7 \%)$ & $\pm 7,7$ & (22.2\%) & $(77,8 \%)$ & $(51,8 \%)$ & $(48,1 \%)$ & $(14,8 \%)$ & $(85,2 \%)$ & $(55,6 \%)$ & $(44,4 \%)$ \\
\hline $3^{\circ}$. & 36 & 22,1 & 8 & 28 & 17 & 19 & 3 & 33 & 19 & 17 \\
\hline ano & $(34,3 \%)$ & $\pm 6,7$ & $(22,2 \%)$ & $(77,8 \%)$ & $(47,2 \%)$ & $(52,8 \%)$ & $(8,3 \%)$ & $(91,7 \%)$ & $(52,8 \%)$ & $(47,2 \%)$ \\
\hline $4^{\circ}$ & 30 & 23,7 & 6 & 24 & 18 & 12 & 4 & 26 & 16 & 14 \\
\hline ano & $(28,6 \%)$ & $\pm 3,6$ & $(20 \%)$ & $(80 \%)$ & $(60 \%)$ & $(40 \%)$ & $(13,3 \%)$ & $(86,7 \%)$ & (53.3\%) & $(46,7 \%)$ \\
\hline & 105 & 22,5 & 24 & 81 & 56 & 49 & 13 & 92 & 53 & 52 \\
\hline Total & $(100 \%)$ & $\pm 6,5$ & $(22,9 \%)$ & (77.1\%) & $(53,3 \%)$ & $(46,7 \%)$ & $(12,4 \%)$ & $(87,6 \%)$ & $(49.5 \%)$ & $(49,5 \%)$ \\
\hline
\end{tabular}

Análise descritiva

Legenda: $\mathrm{n}=$ número de participantes; \% = percentual; $\mathrm{DP}=$ desvio padrão; $\mathrm{M}=$ masculino; $\mathrm{F}=$ feminino 
Tabela 2. Perfil do estresse dos discentes participantes

\begin{tabular}{|c|c|c|c|c|c|c|c|c|}
\hline & \multirow{3}{*}{$\begin{array}{c}\text { Com } \\
\text { estresse }\end{array}$} & \multicolumn{4}{|c|}{ Fase do estresse } & \multicolumn{3}{|c|}{ Predominância de sintomas } \\
\hline & & $\begin{array}{l}\text { Fase do } \\
\text { alerta }\end{array}$ & $\begin{array}{l}\text { Fase da } \\
\text { resistência }\end{array}$ & $\begin{array}{c}\text { Fase de } \\
\text { quase } \\
\text { exaustão }\end{array}$ & $\begin{array}{l}\text { Fase de } \\
\text { exaustão }\end{array}$ & Físico & Psicológico & $\begin{array}{c}\text { Físico e } \\
\text { Psicológico }\end{array}$ \\
\hline & & $\mathrm{n}(\%)$ & $\mathrm{n}(\%)$ & $\mathrm{n}(\%)$ & n (\%) & $\mathrm{n}(\%)$ & $\mathrm{n}(\%)$ & $\mathrm{n}(\%)$ \\
\hline \multirow{2}{*}{$1^{\circ}$. ano } & 4 & 0 & 4 & 0 & 0 & 1 & 3 & 0 \\
\hline & $(33,3 \%)$ & $(0 \%)$ & $(100 \%)$ & $(0 \%)$ & $(0 \%)$ & $(25 \%)$ & $(75 \%)$ & $(0 \%)$ \\
\hline \multirow{2}{*}{$2^{\circ}$. ano } & 24 & 0 & 18 & 6 & 0 & 7 & 13 & 4 \\
\hline & $(88,9 \%)$ & $(0 \%)$ & $(75 \%)$ & $(25 \%)$ & $(0 \%)$ & $(29,2 \%)$ & $(54,2 \%)$ & $(16,7 \%)$ \\
\hline \multirow{2}{*}{$3^{\circ}$. ano } & 27 & 1 & 23 & 3 & 0 & 4 & 19 & 4 \\
\hline & $(75 \%)$ & $(3,7 \%)$ & $(85,2 \%)$ & $(11.1 \%)$ & $(0 \%)$ & $(14,8 \%)$ & $(70,4 \%)$ & $(14,8 \%)$ \\
\hline \multirow{2}{*}{$4^{\circ}$. ano } & 26 & 1 & 18 & 6 & 1 & 3 & 17 & 6 \\
\hline & $(86,7 \%)$ & $(3,8 \%)$ & $(69,2 \%)$ & $(23,1 \%)$ & $(3,8 \%)$ & $(11,5 \%)$ & $(65,4 \%)$ & $(23,1 \%)$ \\
\hline
\end{tabular}

Análise descritiva

Legenda: $\mathrm{n}$ = número de participantes; \% = percentual

Tabela 3. Associação entre a presença de estresse, o sexo, a formação no ensino médio, exercício de atividade remunerada e recebimento de bolsa e/ou auxílio da universidade

\begin{tabular}{|c|c|c|c|c|c|c|c|c|}
\hline & \multicolumn{2}{|c|}{ Sexo } & \multicolumn{2}{|c|}{$\begin{array}{c}\text { Formação no ensino } \\
\text { médio }\end{array}$} & \multicolumn{2}{|c|}{ Atividade remunerada } & \multicolumn{2}{|c|}{$\begin{array}{c}\text { Bolsa ou auxílio da } \\
\text { universidade }\end{array}$} \\
\hline & M & $\mathbf{F}$ & Pública & Privada & Não & Sim & Não & Sim \\
\hline Sem estresse & $\begin{array}{c}10 \\
(41,7 \%)\end{array}$ & $\begin{array}{c}14 \\
(17,3 \%)\end{array}$ & $\begin{array}{c}14 \\
(25,9 \%)\end{array}$ & $\begin{array}{c}10 \\
(19,6 \%)\end{array}$ & $\begin{array}{c}18 \\
(19,6 \%)\end{array}$ & $\begin{array}{c}6 \\
(46,1 \%)\end{array}$ & $\begin{array}{c}15 \\
(28,9 \%)\end{array}$ & $\begin{array}{c}9 \\
(16,9 \%)\end{array}$ \\
\hline Com estresse & $\begin{array}{c}14 \\
(58,3 \%)\end{array}$ & $\begin{array}{c}67 \\
(82,7 \%)\end{array}$ & $\begin{array}{c}40 \\
(74,1 \%)\end{array}$ & $\begin{array}{c}41 \\
(80,4 \%)\end{array}$ & $\begin{array}{c}74 \\
(80,4 \%)\end{array}$ & $\begin{array}{c}7 \\
(53,9 \%)\end{array}$ & $\begin{array}{c}37 \\
(71,1 \%)\end{array}$ & $\begin{array}{c}44 \\
(83,1 \%)\end{array}$ \\
\hline valor de $p$ & \multicolumn{2}{|c|}{$0,01^{*}$} & \multicolumn{2}{|c|}{0,40} & \multicolumn{2}{|c|}{$0,03^{* *}$} & \multicolumn{2}{|c|}{0,10} \\
\hline
\end{tabular}

*valores significativos $(p<0,05)$ de acordo com o teste Qui-quadrado

Legenda: $M=$ masculino; $F=$ feminino

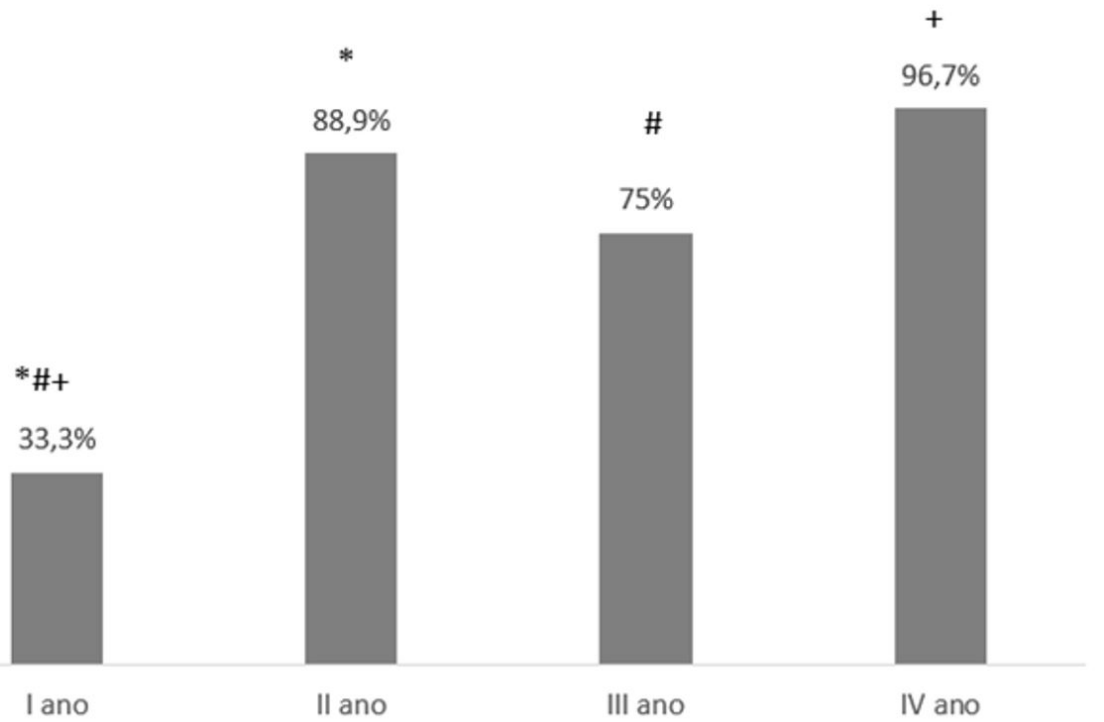

Figura 1. Influência do ano de estudo no número de pessoas com estresse

Teste anova de uma via e Teste post Hoc de Tukey; * valores significativos entre o I e II ano (p-valor $<0,01)$; \# entre I e III $(\mathbf{p}$-valor $<0,01)$ e + entre I e IV ( $p$-valor $<0,01)$

Foi evidenciada a presença de estresse em 77,1\% (81) dos discentes, com prevalência da fase de "resistência" e de sintomas psicológicos (Tabela 2).

Houve efeito do ano estudado na presença de estresse $\left(F_{3,101}=9,825 ; p<0,01\right)$ e diferença entre o primeiro ano e os demais ( $<<0,01$ para todas as comparações) (Figura 1).

Os resultados indicaram associação do estresse com o sexo e o fato de não exercer atividade remunerada (Tabela 3).

\section{DISCUSSÃO}

Os resultados deste estudo apontaram um número elevado de discentes da graduação em Fonoaudiologia com estresse, sendo a prevalência superior à encontrada em outros estudos com universitários da área da saúde ${ }^{(5-8)}$ e muito superior, quando comparada a de estudantes de outras áreas ${ }^{(1,12)}$. 
Destaca-se que os questionários nesta pesquisa foram aplicados no fim de um semestre letivo, o que pode ter influenciado os altos índices de estresse encontrados, em relação àqueles que poderiam ser observados caso os questionários fossem aplicados no início do semestre, visto que o final de semestre é o período com grande concentração de provas e atividades que precisam ser finalizadas, portanto, com maior presença de eventos estressores.

Referente à análise da fase do estresse em que os discentes se encontravam, constatou-se predominância da fase de "resistência", seguida pela fase de "quase exaustão", assim como observado em estudos semelhantes ${ }^{(5-8)}$. Os resultados indicaram, também, que os discentes investigados apresentaram, principalmente, recursos psicológicos para lidar com o estresse, relação que pode ser justificada pelo fato de que a fase de "resistência" é a fase de reequilíbrio físico e mental, sendo a ansiedade e isolamento social uns de seus principais sintomas ${ }^{(13)}$.

Em relação ao sexo, o estresse foi significativamente maior entre as mulheres, achado semelhante ao de outros estudos ${ }^{(1,9,13)}$ que consideraram que os eventos estressores enfrentados por universitários de ambos os sexos são basicamente os mesmos e que o perfil dessa população não indica sobrecarga de trabalho e dos cuidados com a família, sugerindo que a diferença no nível de estresse pode ser de origem biológica, cognitiva e comportamental, associada a cobranças sociais em relação à mulher, quando as práticas parentais podem gerar maior nível de estresse. Esses são fatores que precisam ser mais bem estudados e ressalta-se que a amostra do presente estudo não foi semelhante entre os sexos, o que pode ter sido um viés que contribuiu para que as mulheres fossem apontadas como mais estressadas.

Outro achado relevante desta pesquisa foi a presença mais significativa de estresse entre os discentes que não trabalhavam. Diferentemente, um estudo com discentes de um curso de Medicina $^{(14)}$ observou maior estresse entre os discentes que trabalhavam, justificando o fato de que o trabalho, por si só, é uma importante fonte de estresse. A diferença pode estar relacionada a um nível socioeconômico, geralmente inferior, dos estudantes de Fonoaudiologia, indicando que as dificuldades financeiras são importante geradoras de estresse durante o curso, maiores, inclusive, que o fato de trabalhar. Entretanto, a baixa frequência de pessoas que exerciam atividades remuneradas pode ter interferido nos resultados.

Quanto ao período mais estressante durante os cursos de graduação, os estudos são controversos. Neste estudo, estar matriculado no primeiro ano foi um fator significativamente menos estressante, se comparado aos demais. Além disso, foi observado que os picos de estresse se concentravam no segundo e no quarto ano, o que pode ser justificado pelo fato de que é no segundo ano do curso que o discente passa a ter contato com matérias específicas da prática fonoaudiológica, trazendo mais responsabilidade, assim como o compromisso com a retenção de conteúdos que serão essenciais para a prática profissional. Já no quarto ano, a maior prevalência de estresse pode ser justificada pelo aumento dos compromissos e responsabilidades, com o contato direto com os pacientes nos estágios, a elaboração do trabalho de conclusão de curso e a expectativa diante da empregabilidade ao fim da graduação ${ }^{(7)}$.

Contrariamente, em um estudo com alunos de Medicina ${ }^{(7)}$, os primeiros anos foram considerados mais estressantes devido à necessidade de adaptação à vida universitária, ao alto número de disciplinas teóricas, à responsabilidade de articular o conteúdo com as práticas clínicas futuras e ao autopoliciamento, com o desenvolvimento inicial da graduação, sugerindo que, com o passar do tempo, os discentes desenvolveram estratégias de enfrentamento diante da nova situação acadêmica ${ }^{(7)}$.

Os estudos são unânimes quanto à diversidade de fatores que podem ser desencadeantes de estresse em universitários e, por não ter sido o objetivo do presente estudo verificar os possíveis agentes estressores e as ações de enfrentamento do estresse utilizadas pelos discentes, são necessários novos estudos com esse objetivo.

Os resultados observados, entretanto, constituem um avanço importante para a Fonoaudiologia, ao indicarem a necessidade de repensar o processo de formação, buscando um monitoramento permanente e o fomento de ações universitárias voltadas para a promoção de saúde dos discentes e de um ambiente acadêmico saudável e benéfico para a saúde mental dessa população e, consequentemente, para a qualidade de vida do futuro fonoaudiólogo.

\section{CONCLUSÃO}

Identificou-se um elevado número de discentes de Fonoaudiologia com estresse, sendo a fase de "resistência" a mais frequente, com a prevalência de sintomas psicológicos. Os fatores associados ao estresse foram sexo, com maior prejuízo entre as mulheres, o fato de os discentes não exercerem atividade remunerada e o ano do curso em que estavam matriculados, sendo o primeiro ano com menor prevalência de estresse e o último ano, com a maior.

\section{REFERÊNCIAS}

1. Lameu JN, Salazar TL, Souza WF. Prevalência de sintomas de stress entre graduandos de uma universidade pública. Psicol Educ. 2016;42:13-22

2. Rovida TAS, Sumida DH, Santos AS, Moimaz SAS, Garbin CAS. Estresse e o estilo de vida dos acadêmicos ingressantes em um curso de graduação em Odontologia. Rev ABENO. 2015;15(3):26-34. http:// dx.doi.org/10.30979/rev.abeno.v15i3.193.

3. Selye H. The stress of life. New York: McGraw Hill; 1984.

4. Lipp MEN. Manual do inventário de sintomas de stress para adultos de Lipp. São Paulo: Casa do Psicólogo; 2005.

5. Torquato JA, Goulart AG, Vicentin P, Correa U. Avaliação do estresse em estudantes universitários. InterSciencePlace. 2010;3(14):140-4.

6. Murakami K, Panúncio-Pinto MP, Santos J, Troncon LE. Estresse psicológico em estudantes de cursos de graduação da área da saúde. Rev Med (São Paulo). 2019;98(2):108-13. http://dx.doi.org/10.11606/ issn.1679-9836.v98i2p108-113.

7. Kam SXL, Toledo ALS, Pacheco CC, Souza GFB, Santana VLM, Bonfá-Araujo B, et al. Estresse em estudantes ao longo da graduação médica. Rev Bras Educ Med. 2019;43(1):246-53. http://dx.doi. org/10.1590/1981-5271v43suplemento1-20180192.

8. Lima RL, Soares MEC, Prado SN, Albuquerque GSC. Estresse do estudante de medicina e rendimento acadêmico. Rev Bras Educ Med. 2016;40(4):678-84. http://dx.doi.org/10.1590/1981$52712015 \mathrm{v} 40 \mathrm{n} 4 \mathrm{e} 01532015$. 
9. Borine RCC, Wanderley KS, Bassitt DP. Relação entre a qualidade de vida e o estresse em acadêmicos da área da saúde. Est Inte Psicol. 2015;6(1):100-18. http://dx.doi.org/10.5433/2236-6407.2015v6n1p100.

10. Oliveira JT, Bittencourt ZZLC. Atividades extracurriculares e qualidade de vida de alunos de Fonoaudiologia. Serv Soc \& Saúde. 2015;13(1):141-50. http://dx.doi.org/10.20396/sss.v13i1.8634926.

11. Costa LBS. Avaliação do estresse e do o rendimento acadêmico em estudantes da área da saúde da Universidade de Brasília [dissertação]. Brasília: Universidade de Brasília; 2018.
12. Padovani RC, Neufeld CB, Maltoni J, Barbosa LNF, Souza WF, Cavalcanti HAF, Lameu JN. Vulnerabilidade e bem-estar psicológicos do estudante universitário. Rev Bras Ter Cogn. 2014;10(1):2-10.

13. Calais SL, Andrade LM, Lipp ME. Diferenças de sexo e escolaridade na manifestação de stress em adultos jovens. Psicol Reflex Crit. 2003;16(2):257-63. http://dx.doi.org/10.1590/S0102-79722003000200005.

14. Marchini DMF, Cardoso R, Oswaldo Y, Pires SRI. Análise de estresse e qualidade de vida em alunos universitários. RAU - Rev Adm Unimep. 2019;17(3):141-64. 